Andrie Jeremy Formanez, MD

Department of Otorhinolaryngology Head and Neck Surgery

Armed Forces of the Philippines Medical Center Quezon City, Philippines

Correspondence: Dr. Andrie Jeremy Formanez Department of Otorhinolaryngology-Head and Neck Surgery Armed Forces of the Philippines Medical Center 7th Floor Armed Forces of the Philippines Medical Center

V. Luna Avenue, Quezon City 0840 Philippines

Phone: (632) 4262701 local 6172

Email: docdrie@yahoo.com

The author declared that this represents original material, that the manuscript has been read and approved by the author, that the requirements for authorship have been met by the author, and that the author believes that the manuscript represents honest work.

Disclosures: The author signed a disclosure that there are no financial or other (including personal) relationships, intellectual passion, political or religious beliefs, and institutional affiliations that might lead to a conflict of interest

\section{Vocal Fold Paralysis with Intraoperative Recurrent Laryngeal Nerve Identification versus Non-Identification of Recurrent Laryngeal Nerve in Total Thyroidectomy: A Retrospective Cohort Study}

\begin{abstract}
Objective: To determine the risk of vocal fold paralysis in patients who underwent total thyroidectomy with and without intraoperative recurrent laryngeal nerve identification.
\end{abstract}

\section{Methods:}

\section{Design: Retrospective Cohort Study}

Setting: Tertiary Military Hospital

Participants: Two hundred thirty seven (237) adult patients who underwent total thyroidectomy for benign lesions based on post-operative histopathology operated on by senior third or fourth year residents. Excluded were those who underwent lobectomy with isthmusectomy or reoperation/completion thyroidectomy, had intrathoracic goiters, confirmed malignancies based on post-operative histopathology, or cases wherein the RLN had to be sacrificed due to gross involvement of the nerve caused by malignancy.

Results: Group A, wherein intraoperative identification of RLN was done, had a temporary and permanent RLN injury incidence of $2.75 \%$ and $0.92 \%$ respectively. Group B, wherein intraoperative identification of RLN was not done, had a temporary and permanent RLN injury incidence of $17.19 \%$ and $12.5 \%$, respectively. Through binary linear regression, the probability of having temporary paralysis increases almost two-fold if the nerve is not identified, and the probability of having permanent paralysis increases by almost nine-fold if the nerve is not identified.

Conclusion: We recommend routine intraoperative RLN identification, which has a lower risk for temporary and permanent vocal fold paralysis when compared to non-identification of the RLN.

Keywords: cranial nerve injuries/prevention and control, recurrent laryngeal nerve injuries, thyroid neoplasms/surgery, thyroidectomy/adverse effects, vocal cord paralysis/prevention control 
latrogenic recurrent laryngeal nerve (RLN) injury remains one of the most common complications of thyroid surgery. Temporary RLN injury occurs in $2.5 \%$ to $5 \%$ of thyroidectomy patients of complications following thyroidectomy. ${ }^{1}$ Permanent RLN injury occurs in approximately $1 \%$ to $1.5 \%$ of total thyroidectomy procedures.' Patients with this complication suffer from different manifestations, the least serious of which is hoarseness. However, this can be particularly devastating in patients for whom the quality of their voice is integral to their occupation. ${ }^{2}$ More serious manifestations include aspiration and dyspnea, which are potentially life-threatening. ${ }^{3}$ Further hospitalization is required to address these problems, which would have been unnecessary had the integrity of the RLN been preserved.

The risk factors that contribute to morbidity following thyroid surgery are well-defined, but their actual contribution is still open to question. ${ }^{4}$ Increased extent of dissection, surgeon experience, malignancy, underlying thyroid disease, and intraoperative technique have been shown to affect post-thyroidectomy morbidity. ${ }^{5}$ In addition, operation for completion ${ }^{6}$ and addition of neck dissection ${ }^{7}$ are additional risk factors for postoperative thyroid morbidity. Surgical technique, more specifically intraoperative RLN identification, is one of the factors which may affect the outcome of thyroid surgery, but some surgeons still do not routinely identify the RLN as can be gleaned from studies in India, ${ }^{8}$ Turkey, ${ }^{9}$ and China. ${ }^{10}$

In our institution, the Department of Otorhinolaryngology - Head and Neck Surgery receives 3 - 5 referrals for hoarseness following thyroid surgery annually. This number is significant given the number of thyroid surgeries done per year and gives cause for alarm given the potentially catastrophic complications of RLN injury. The significant number of referrals warrants investigation of the possible causes. Furthermore, to the best of our knowledge, no local data has been published regarding the incidence and causative factors of RLN injury following thyroidectomy. Hence, the purpose of this study is to determine the risk of vocal fold paralysis in patients who underwent total thyroidectomy with and without recurrent laryngeal nerve identification.

\section{METHODS}

With Ethical Review Board (ERB) approval, this retrospective cohort study consisted of 237 adult patients who underwent total thyroidectomy and presented with benign lesions based on postoperative histopathology from January 2009 to December 2014 at the V. Luna General Hospital, a tertiary military hospital. Operations were performed by senior third year or fourth year residents.

Patients who underwent only lobectomy with isthmusectomy or underwent reoperation/completion thyroidectomy were excluded from the study. Patients who presented with prior vocal fold paralysis, intrathoracic goiters, or with suspicious/confirmed malignancies based on post-operative histopathology were also excluded. Furthermore, cases wherein the RLN had to be sacrificed due to gross involvement of the nerve by malignancy were excluded. Preoperative evaluation of the patients included thyroid ultrasound, free T4 and thyroid stimulating hormone determination, serum calcium concentration, fine-needle aspiration biopsy and evaluation of vocal fold mobility by flexible or rigid laryngoscopy.

Patients were divided into two groups based on a review of operative techniques in their records. Patients in Group A were those wherein the surgeon indicated that he/she identified and preserved the RLN during the procedure whereas patients in Group B were those where the surgeon did not indicate that he/she identified or preserved the RLN. Intraoperative dimensions of the thyroid tumors were also recorded.

Records of flexible nasopharyngolaryngoscopy or rigid laryngoendoscopy performed by resident physicians of the Department of ORL -HNS on patients who manifested with hoarseness post-operatively were reviewed. Vocal fold paralysis was defined as having decreased or absent vocal fold mobility upon assessment. (Figure 1) Patients thus assessed were then followed-up regularly at three-month intervals for the first post-operative year. Permanent vocal fold paralysis was diagnosed when vocal fold mobility did not return 6 months after surgery.

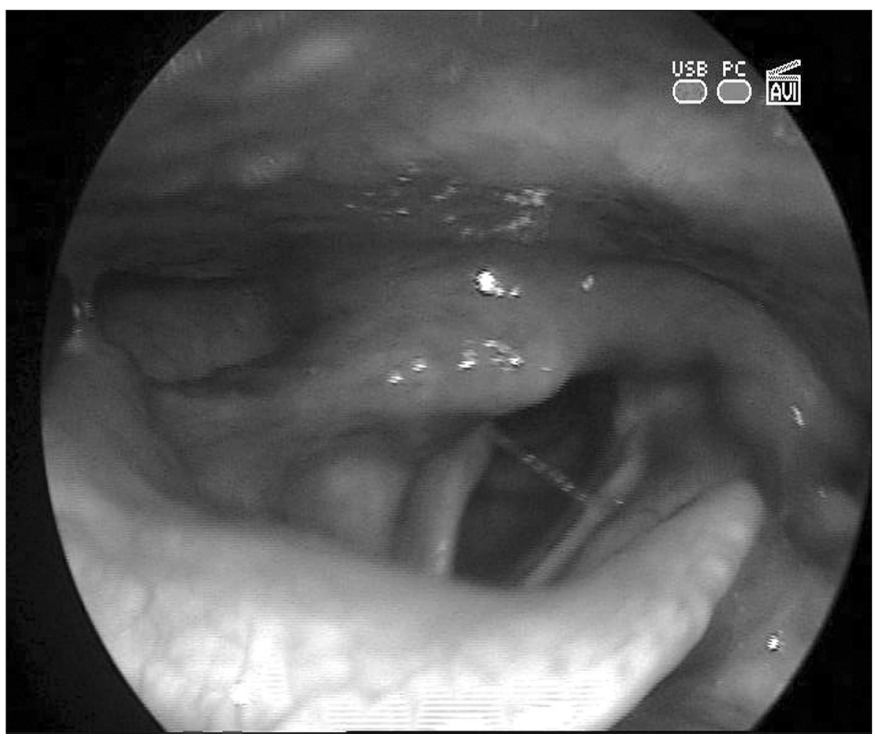

Figure 1. Still Photo of the Glottis and Supraglottis of a Patient with Paralysis of Right Recurrent Laryngeal Nerve following Total Thyroidectomy 


\section{ORIGINAL ARTICLES}

Age, sex, tumor size, temporary and permanent post-operative vocal cord paralysis were tabulated using Microsoft Office Excel 2007 (Microsoft Corp., Washington, USA). IBM SPSS 23 (International Business Machines Corp.) was used for the independent T-test and binary logistic regression analysis.

The prevalence of temporary and recurrent vocal fold paralysis were expressed as percentages of the total number of cases per group. Independent T-test was used to determine if there was a significant difference in tumor size between both groups. Binary logistic regression analysis was used to evaluate the relationship between intraoperative RLN identification and RLN preservation.

\section{RESULTS}

A total of 237 patients with an age range of 20 to 65 years old (median age 41 years old) were included. There were more females than males (163:74). There were 109 patients in Group A, wherein 3 demonstrated temporary RLN injury with an incidence of $2.75 \%$. The RLN injuries in all these 3 patients were unilateral. Furthermore, the average tumor size in these patients taken as the widest diameter was at $5.6 \pm 0.93 \mathrm{~cm}$. In comparison, there were 128 patients in Group $B$ wherein 22 patients presented with temporary RLN injury and an incidence $17.19 \%$. (Table 1) Of these patients, 3 had bilateral RLN injury. Average tumor size in this group was $5.3 \pm 0.78 \mathrm{~cm}$. There was no significant difference in tumor size between the two groups based on independent T-Test at 95\% confidence interval $(p=1.96)$.

Table 1. Incidence of Temporary and Permanent RLN Paralysis

\begin{tabular}{|l|c|c|} 
& Group A & Group B \\
\hline Number of Patients & 109 & 128 \\
\hline Patients with Temporary Paralysis & 3 & 22 \\
\hline Incidence of Temporary Paralysis & $2.75 \%$ & $17.19 \%$ \\
\hline Patients with Permanent Paralysis & 1 & 16 \\
\hline Incidence of Permanent Paralysis & $0.92 \%$ & $12.50 \%$ \\
\hline Bilateral RLN injury & 0 & 3 \\
\hline
\end{tabular}

Through binary linear regression, the odds of having temporary paralysis in Group A over Group B was found to be $e^{-0.6819142}=0.5056$. Its reciprocal 1.98 means that the probability of having temporary paralysis increases by almost two-fold if the nerve is not identified.

There was only one patient with permanent RLN injury in Group A, with an incidence of $0.92 \%$. In contrast, there were 16 patients in Group $B$ who had permanent RLN injury, and an incidence of $12.5 \%$. Of these, 2 had bilateral RLN injury. The odds of having permanent paralysis for
Group A over Group B was found to be $e^{-2.158925}=0.1154$. Obtaining its reciprocal leads to 8.67 , meaning that the probability of having permanent paralysis increases by almost nine-fold if not identified.

\section{DISCUSSION}

This study found that intraoperative RLN identification has a lower risk for temporary and permanent vocal cord paralysis compared to non-identification of the RLN. The importance of preservation of the RLN cannot be overemphasized. In agreement with Pradeep et al., our findings suggest that the key to achieving preservation is to trace the entire course of the nerve along the tracheoesophageal groove up to its entry into the larynx. ${ }^{9}$

In this study, confounding factors which may cause or contribute to vocal cord paralysis such as malignancy, reoperation, completion thyroidectomy, were taken out of the equation based on inclusion and exclusion criteria. Tumor size, which is another confounding factor, was not statistically significant, with similar tumor sizes in both groups.

However, since this was a retrospective cohort study, it was not able to control for the uniformity of the entire operative technique as well as the skill of the surgeon. Since the study was done at a training institution, the experience of senior residents is limited to the number of cases to which they are exposed. Post-hoc analysis revealed that third year surgeons accounted for $67 \%$ of temporary paralysis in group $A$ while a fourth year surgeon was responsible for the remaining case. In group B, third year surgeons accounted for $45 \%$ of temporary paralysis compared to the $55 \%$ of cases handled by fourth year surgeons. The lone case of permanent paralysis in group $A$ was performed by a fourth year surgeon. In group B, 75\% of the cases of permanent paralysis were handled by third year surgeons, compared to the $25 \%$ which were handled by fourth year surgeons. This may suggest that experience does play a role in cases when the recurrent nerve was not routinely identified.

The results of this study showed less temporary and permanent vocal cord paralysis in patients where intraoperative RLN identification was performed. Moreover, statistical analysis using binary linear regression confirms that intraoperative RLN identification serves as a protective factor against temporary and permanent RLN injury following thyroid surgery as evidenced by the increased probability of RLN injury if the nerve was not identified during surgery.

The prevalence of temporary and permanent vocal paralysis for group $A$ at $2.75 \%$ and $0.92 \%$, respectively are slightly higher when compared to the finding of Canbaz et al. and Veyseller et al. who reported no paralysis for the group where the RLN was preserved and identified. ${ }^{9,10}$ 
Likewise, the rates of temporary and permanent vocal cord paralysis in group B at $17.19 \%$ and $12.15 \%$, respectively, are higher than those of the two aforementioned studies $(0 \%, 7.9 \%)$ and $(1 \%, 1.5 \%)$. The higher incidences of temporary and permanent RLN injury in this study may be attributed to the fact that the surgeons in group A only followed the main trunk of the RLN up to its entry into the larynx while the surgeons in group B did not identify the RLN as compared to the previous studies where the RLN and its branches were meticulously followed up to its entry to the larynx. ${ }^{9,10}$ Injury to the RLN can be brought about by inadvertent transection of a bifurcation of the nerve prior to its entry into the larynx, this bifurcation being present $34 \%-70 \%$ of the time..$^{11,12}$

A considerable number of surgeons do not perform routine intraoperative RLN identification since it is said to cause hypoparathyroidism, mainly through devascularization of the parathyroid glands. ${ }^{13}$ However, among experienced surgeons who routinely identify the RLN, temporary and permanent hypoparathyroidism was not high, with incidences of $9.6 \%$ and $0.7 \%$, respectively. ${ }^{14}$

It can be concluded from this study that intraoperative RLN identification has a lower risk for temporary and permanent vocal cord paralysis when compared to non-identification of the RLN. The probability of having temporary paralysis increases almost two-fold if the nerve is not identified, and the probability of having permanent paralysis increases by almost nine-fold if the nerve is not identified. While future studies may assess RLN injury and other complications of thyroidectomy by comparing surgeons who routinely perform intraoperative RLN identification and others who do not, we recommend routine identification of the RLN.
REFERENCES
1. Lai SY, Mandel SJ, Weber RS. Management of Thyroid Neoplasms. In: Flint PW, Haughey BH, Lund VJ, Niparko JK, Richardson MA, Robbins KT, et al, editors. Cummings Otolaryngology Head and Neck Surgery. Philadelphia: Mosby-Elsevier; 2010. p. 1770 - 1771.
2. Zakaria HM, Alwad NA, Kreedes AS, Al-Mulhim AM, Al-Sharway MA, Hadi MA, et al. Recurrent laryngeal nerve injury in thyroid surgery. Oman Med J. 2011 Jan; 26(1): 34-38.
3. Bergamaschi R, Becouarn G, Ronceray J, Arnaud JP. Morbidity of thyroid surgery. Am J Surg. 1998 Jul; 176(1):71-75.
4. Dackiw APB, Rotstein LE, Clark OH. Computer-assisted evoked electromyography with stimulating surgical instruments for recurrent/external laryngeal nerve identification and preservation in thyroid and parathyroid operation. Surgery. 2002 Dec; 132(6):1100-1108.
5. Thomusch O, Sekulla C, Dralle H. Is primary total thyroidectomy justified in benign multinodular goiter? Results of a prospective quality assurance study of 45 hospitals offering different levels of care. Chirurg. 2003 May; 74(5):437-443.
6. Shaha AR, Jaffe BM. Completion thyroidectomy: a critical appraisal. Surgery. 1992 Dec: 112(6):1148-1152.
7. Betka J, Mrzena L, Astl J, Nemec J, Vlcek P, Taudy M, Skrivan J. Surgical treatment strategy for thyroid gland carcinoma nodal metastases. Eur Arch Otorhinolaryngol. 1997; 254(s1):169-174.
8. Pradeep PV, Jayashree B, Harshita S. A Closer Look at Laryngeal Nerves during Thyroid Surgery: A Descriptive Study of 584 Nerves. Anat Res Int. 2012; 2012: 490390, 6 pages. Available from http://dx.doi.org/10.1155/2012/490390
9. Veyseller B, Aksoy F, Selim Y, Karatas A, Ozturan O. Effect of Recurrent Laryngeal Nerve Identification Technique in Thyroidectomy on Recurrent Laryngeal Nerve Paralysis and Hypoparathyroidism. Arch Otolaryngol Head Neck Surg. 2011 Sep; 137(9):897-900.
10. Canbaz H, Dirlik M, Colak T, Ocal K, Akca T, Bilgin O, et al. Total thyroidectomy is safer with identification of recurrent laryngeal nerve. J Zhejiang Univ Sci B. 2008 Jun; 9(6): 482-488.
11. Kandil E, Abel Khalek M, Aslam R, Friendlander P, Bellows CF, Slakey D. Recurrent laryngea nerve: signifance of the anterior extralaryngeal branch. Surgery 2011 Jun: 149(6):820-824.
12. Pascoal AAF, Fernandes JR, Ruiz CR, Person OC, Nascimento SRR. Terminal Branch of Recurrent Laryngeal Nerve. Advances in Anatomy Volume 2014, Article ID 858539, 5 pages, 2014.
13. Megherbi MT, Graba A, Abid L, Oulmane D, Saidani M, Benabadji R. Complications and sequela of benign thyroid surgery. J Chir (Paris) 1992 Jan; 129(1):41-46.
14. Zambudio AR, Rodriguez J, Riquelme J, Soria T, Canteras M, Parrilla P. Prospective study of postoperative complications after total thyroidectomy for multinodular goiters by surgeons with experience in endocrine surgery. Ann Surg. 2004 Jul; 240(1):18-25.

